\title{
gु \\ Theoretical proposal for superconducting spin qubits
}

\author{
C. Padurariu and Yu. V. Nazarov \\ Kavli Institute of NanoScience, Delft University of Technology, Lorentzweg 1, 2628 CJ Delft, The Netherlands
}

(Received 20 December 2009; revised manuscript received 5 April 2010; published 29 April 2010)

\begin{abstract}
We propose and theoretically investigate superconducting spin qubits. Superconducting spin qubit consists of a single spin confined in a Josephson junction. We show that owing to spin-orbit interaction, superconducting phase difference across the junction can polarize this spin. We demonstrate that this enables single-qubit operations and more complicated quantum gates, where spins of different qubits interact via a mutual inductance of the superconducting loop where the junctions are embedded. Recent experimental realizations of Josephson junctions made of semiconductor quantum dots in contact with superconducting leads have shown that the number of electrons in the quantum dot can be tuned by a gate voltage. Superconducting spin qubit is realized when the number of electrons is odd. We discuss the qubit properties at phenomenological level. We present a microscopic theory that enables us to make accurate estimations of the qubit parameters by evaluating the spin-dependent Josephson energy in the framework of fourth-order perturbation theory.
\end{abstract}

DOI: 10.1103/PhysRevB.81.144519

PACS number(s): 74.50.+r, 73.63.Kv, 85.25.Cp, 03.67.Lx

\section{INTRODUCTION}

Potential benefits of a quantum computer: secure communication, fast database searching, and efficient prime factorization $^{1-3}$ have inspired significant research efforts. Building a quantum computer requires the realization of qubits as its elementary units. Useful qubits should satisfy two conditions: they can be manipulated and read before the quantum information stored in their state is lost, and they allow for engineering of a controllable interaction between them. Designing and realizing such qubits defines the focus of most research in the field.

Since the electron spin provides the simplest example of a coherent two-level system, that is, a qubit, spin-based qubits very soon became a subject of intense theoretical ${ }^{4}$ and experimental research. Experiments proved relatively long $T_{1}$ and $T_{2}$ times for single-electron spins trapped in quantum dots, ${ }^{5-9}$ diamond, ${ }^{10-13}$ and other materials. Good isolation from the environment protects from decoherence at cost of hampering qubit control and readout. While current research successfully addresses these shortcomings, ${ }^{14-17}$ the realization of controllable interaction between pairs of spin qubits has been so far obstructed by numerous practical problems. ${ }^{18}$

Superconducting qubits do better in this respect. Quantum logic gates involving the controllable interaction of two qubits have been demonstrated in a variety of setups. ${ }^{19-23} \mathrm{Su}-$ perconducting qubits exploit Josephson effect and Coulomb blockade, both immediately related to electric variables of flux and charge. This allows for easy integration of qubits into electric circuits and is the reason of the better performance. At the same time, this forms the stumbling block on the way to a superconducting quantum computer: the sensitivity to noise of the electric variables ${ }^{22,24,25}$ results in relatively short decoherence times.

In this paper, we propose a design of superconducting spin qubit and discuss its feasibility and advantages. A spin is trapped in a quantum dot connected to superconducting leads, thereby forming a Josephson junction, see Fig. 1. Owing to spin-orbit interaction, the superconducting phase difference polarizes the spin. This provides means to read and manipulate its quantum state.
We detail the operation of a single qubit and the design of qubit-qubit interaction that allows to make quantum logic gates. In particular, we emphasize the prospect of all electrical manipulation of the qubit state and qubit-qubit coupling, an important advantage of superconducting spin qubits over other spin qubits. We discuss relaxation and decoherence of superconducting spin qubits, in comparison with other superconducting qubits. We predict that superconducting spin qubits have relatively longer decoherence time and thus, are better suited for use in a quantum computer. To prove feasibility of the qubit design proposed, we present microscopic calculations and numerical estimates of the spin- and gatevoltage-dependent Josephson energy.

Quantum dot connected to superconducting leads is an essential element of our design. Superconducting quantum dots have received theoretical attention rather early. ${ }^{26,27}$ About 10 years later, experimental breakthrough has been achieved by making good contacts between semiconducting nanowires and superconducting leads. ${ }^{28,29}$ Gate electrodes put close to the nanowire can be used to create potential barriers in the nanowire, thereby defining a quantum dot. The quantum dot is in the Coulomb-blockade regime, that is, the number of electrons is tunable. It has been proven that such a dot can be included into a superconducting circuit as a Josephson junction carrying a supercurrent. ${ }^{29}$ The idea of our proposal is to use this setup by keeping odd number of electrons in the dot. In this case, the ground state of the resulting

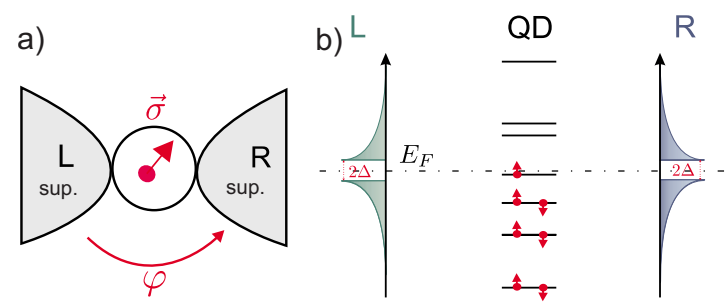

FIG. 1. (Color online) Superconducting spin qubit. (a) Quantum dot with odd number of electrons is connected to superconducting leads ( $\mathrm{L}$ and R) biased at phase difference $\varphi$. (b) Energy levels in quantum dot and the leads. 
Josephson junction is a spin doublet. This is advantageous in comparison to an earlier proposal concerning Andreev quantum dot, ${ }^{30}$ where the spin doublet corresponds to an excited state of the system. Spin-orbit effects in quantum constrictions and dots have been discussed in Refs. 31 and 32.

The structure of the paper is as follows. In Sec. II, we describe the qubit phenomenologically, discussing singlequbit manipulation, design of qubit-qubit interaction, and relaxation and decoherence of the qubit states. Section III focuses on the microscopic description of the Josephson junction with a single spin, leading to expressions of the spin-dependent and spin-independent parts of the Josephson energy in terms of the junction parameters. Section IV discusses different parameter regimes, providing order of magnitude estimations of the Josephson energy. Section V presents numerical calculations of the relevant quantities. Section VI concludes.

\section{PHENOMENOLOGICAL DESCRIPTION}

We proceed with the phenomenological description of the qubit, postponing for now microscopic analysis. An important feature is that the superconducting current flowing through the Josephson junction is determined by the state of the spin enclosed. This can be understood as follows. Junction current is the result of transfer of Cooper pairs between the leads via coherent tunneling events. Conventionally, tunneling events are spin conserving. However, strong spin-orbit coupling mixes the spin states of electrons as they tunnel between orbitals in the superconductor and orbitals in the dot. ${ }^{33}$ The resulting nonspin-conserving tunneling amplitudes depend strongly on the wave function of the levels involved. When a Cooper pair tunnels via two different levels in the dot, the initial spin-singlet configuration acquires complex spin structure, due to the different spin-dependent tunneling amplitudes. If one of the two levels involved is occupied by a single electron, its spin restricts the pathways of Cooperpair tunneling, due to Pauli exclusion. This directly couples current to spin, lifting the degeneracy between spin-up and spin-down states.

\section{A. Effective Hamiltonian}

We describe the spin-polarization effect by the following junction Hamiltonian:

$$
H=E_{j} \cos (\varphi)+\left(\vec{\epsilon}_{\mathrm{so}} \cdot \vec{\sigma}\right) \sin (\varphi),
$$

where $\vec{\sigma}$ is the spin operator. The pseudovector $\vec{\epsilon}_{\text {so }}$ defines the polarization axis in three dimensions. Its magnitude and direction depends on the spin-dependent tunneling amplitudes as well as on the positions of the levels in the quantum dot. As a result, $\vec{\epsilon}_{\mathrm{so}}$ is independent of the superconducting phase difference $\varphi$ but does depend on gate voltage, as the gate electric field modifies quantum-dot wave functions and levels.

We will present estimations of $E_{j}$ and $\vec{\epsilon}_{\mathrm{so}}$ in Sec. IV. For present purposes, it is enough to assume that typical values of $\vec{\epsilon}_{\text {so }}$ are somewhat smaller than $E_{j}$. Actually, $E_{j}$ can be made zero by a certain choice of gate voltages. In the vicinity
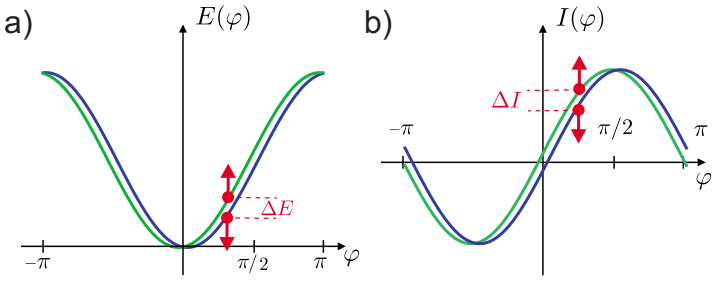

FIG. 2. (Color online) Two states of a simple superconducting circuit [Fig. 3(a)] containing the qubit junction. (a) Energy as a function of phase drop over the qubit junction $\varphi$. (b) Loop current $I(\varphi)$.

of this point, $\vec{\epsilon}_{\mathrm{so}}$ may be bigger than $E_{j}$. However, we do not concentrate on this case.

The junction forms a qubit: it may be found in two spin states, $|\uparrow\rangle$ and $|\downarrow\rangle$, that differ in spin projection along the polarization axis $\vec{\epsilon}_{\mathrm{so}}$. At fixed phase, the energy spacing, $\Delta E=2\left|\vec{\epsilon}_{\mathrm{so}}\right||\sin (\varphi)|$, leads to a measurable difference in superconducting current (Fig. 2),

$$
\Delta I= \pm(2 e / \hbar)\left|\vec{\epsilon}_{\mathrm{so}}\right||\cos (\varphi)| .
$$

The sign is determined by the direction of the spin along $\vec{\epsilon}_{\mathrm{so}}$. This provides the means to read the qubit state.

To illustrate, consider a simple superconducting circuit consisting of a loop interrupted by the qubit junction and a conventional Josephson junction, see Fig. 3(a). We apply magnetic flux through the loop $\phi_{\text {loop }}$. When the Josephson energy of the conventional junction is much larger than the Josephson energy of the qubit $E_{j 2} \gg E_{j 1}$, the phase induced by the magnetic flux is acquired mainly by the qubit junction $\varphi_{2} \approx 0$. The phase difference over the qubit junction can be fixed by fixing the magnetic flux $\varphi_{1} \approx 2 \pi \phi_{\text {loop }} / \phi_{0}, \phi_{0}$ $=\pi \hbar / e$ being the magnetic-flux quantum. The current through the loop is plotted as a function of phase for each of the two states of the qubit (see Fig. 2). The magnetic flux generated by this current can be measured by a nearby superconducting quantum interference device loop, not shown in Fig. 3. This is a common technique used to measure the state of superconducting qubits. ${ }^{24}$
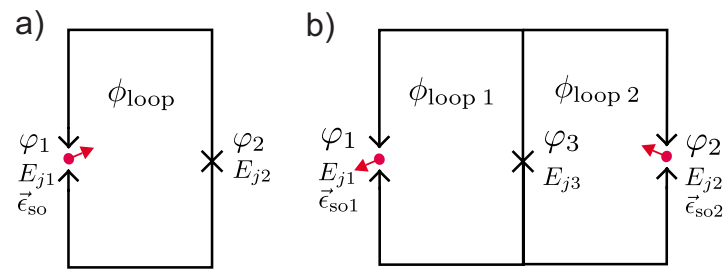

FIG. 3. (Color online) Two simple superconducting circuits. (a) Superconducting loop interrupted by qubit junction (characterized by $E_{j 1}$ and $\vec{\epsilon}_{\mathrm{so}}$ ) and conventional Josephson junction (characterized by $E_{j 2}$ ). The phase of the two junctions are modulated by the magnetic flux, $\varphi_{1}+\varphi_{2}=2 \pi \phi_{\text {loop }} / \phi_{0}$. (b) Two superconducting loops are presented, each interrupted by a qubit junction. The loops have a common side that is interrupted by a conventional Josephson junction $E_{j 3}$. Magnetic flux flowing through the loops is represented by $\phi_{\text {loop1 }}$ and $\phi_{\text {loop2 }}$. 

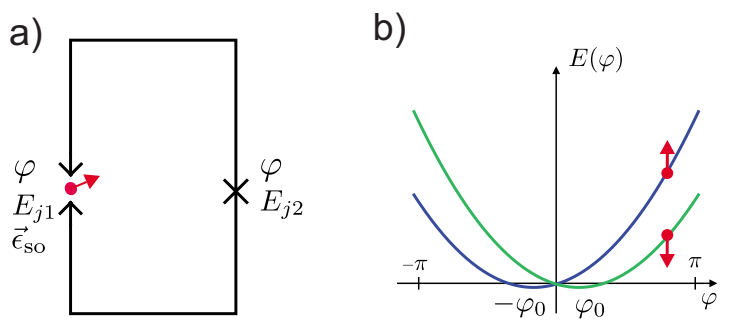

FIG. 4. (Color online) Superconducting loop without magnetic flux. The dependence of energy on phase shows that the minimum energy is achieved at nonvanishing phase $\pm \varphi_{0}$. The sign of the equilibrium phase depends on the orientation of the spin in the qubit junction.

The maximum value of the induced flux is achieved when $E_{j 1} \approx E_{j 2} \approx\left|\vec{\epsilon}_{\mathrm{so}}\right|$ and is on the order of the magnetic-flux quantum $\phi_{0}$. It is interesting to compare the induced flux, with the flux generated by the magnetic dipole moment of the electron spin confined in the quantum dot. Considering an area element perpendicular to the dipole moment and situated at the distance $r$ from it, the flux flowing through the area scales with the distance as $\phi \propto r^{-1}$. We find the distance $r_{0}$ where the magnetic field of a single electron produces a quantum of magnetic flux via the relation $e^{2} /\left(4 \pi \epsilon_{0} r_{0}\right)$ $\approx m_{e} c^{2}$. This distance is well into the subatomic region, on the order of $r_{0} \approx 10^{-15} \mathrm{~m}$.

\section{B. Spontaneous currents}

Let us consider another regime, which is useless for qubit applications but interesting from the point of view of general physics. Let us consider vanishing flux through the loop and assume formula $E_{j 1}, E_{j 2} \ll\left|\vec{\epsilon}_{\text {so }}\right|$. In this case, the phase dependent energy Eq. (1) can be expanded at small $\varphi$,

$$
E(\varphi)=\left(E_{j 1}+E_{j 2}\right) \varphi^{2} / 2 \pm\left|\vec{\epsilon}_{\mathrm{so}}\right| \varphi
$$

with opposite signs corresponding to opposite spin orientations.

We see (Fig. 4) that the equilibrium superconducting phase is nonzero. It takes opposite values depending on spin orientation. The current flowing through the junction is also nonzero $I=E_{j 2} \varphi_{0}$. We stress that at zero flux applied, the system is time reversible. The situation just described can be envisaged as spontaneous breaking of time reversibility. Indeed, the energies of the states with opposite spins are precisely the same. We will present detailed description of the situation elsewhere.

\section{Single-qubit manipulation}

Let us turn our attention to manipulation of the qubit state. It is common to use pulses of an ac field of resonant frequency $\omega=2\left|\vec{\epsilon}_{\text {so }}\right||\sin (\varphi)| / \hbar$. Magnetic fields perpendicular to the loop plane induce modulations of superconducting phase but do not allow resonant manipulation as this does not change $\vec{\epsilon}_{\mathrm{so}}$.

In-plane magnetic fields can polarize qubit spin, deflecting it from the direction of $\overrightarrow{\boldsymbol{\epsilon}}_{\mathrm{so}}$. However, this approach is difficult to realize experimentally due to misalignment. The magnetic field component perpendicular to the loop contributes to the total magnetic flux, changing the properties of the qubit at the same time as the manipulation is performed.

Fortunately, magnetic field is not needed. Rabi oscillations can also be induced electrically via the gate electrodes. We remind that the direction of $\vec{\epsilon}_{\text {so }}$ depends on the position of levels in the dot. Therefore, shifting the gate voltage would also rotate $\vec{\epsilon}_{\mathrm{so}}$. To illustrate, let us assume that a change in the gate voltage leads to a corresponding change in polarization pseudovector $\overrightarrow{\boldsymbol{\epsilon}}_{\mathrm{so}} \rightarrow \overrightarrow{\boldsymbol{\epsilon}}_{\mathrm{so}}+\delta \overrightarrow{\boldsymbol{\epsilon}}_{\mathrm{so}}$. We describe the effect of a resonant gate-voltage pulse by the time-dependent qubit Hamiltonian,

$$
H(t)=\left[\left|\vec{\epsilon}_{\mathrm{so}}\right| \sigma_{z}+\delta \vec{\epsilon}_{\mathrm{so}} \cdot \vec{\sigma} \cos (\omega t)\right] \sin (\varphi),
$$

where the $z$ axis is chosen along $\overrightarrow{\boldsymbol{\epsilon}}_{\text {so. }}$. Assuming $\left|\delta \overrightarrow{\boldsymbol{\epsilon}}_{\text {so }}\right| \ll\left|\overrightarrow{\boldsymbol{\epsilon}}_{\text {so }}\right|$, the dynamics of the qubit is described by Rabi oscillations with frequency,

$$
\hbar \Omega_{R}=\sqrt{\left(\delta \epsilon_{\mathrm{so}, x}\right)^{2}+\left(\delta \epsilon_{\mathrm{so}, y}\right)^{2}}|\sin (\varphi)| .
$$

We stress that if the change $\delta \vec{\epsilon}_{\text {so }}$ is parallel to the initial direction of $\vec{\epsilon}_{\text {so }}$, Rabi oscillations do not occur. In this case, the qubit retains its initial state. Thus, change in $\delta \vec{\epsilon}_{\mathrm{so}}$ in the perpendicular direction is essential for qubit manipulation.

Since electric fields are easier to localize in space than magnetic fields, electrical manipulation is advantageous in view of controlling qubits individually.

\section{Design of qubit-qubit interaction}

Further, let us focus on qubit-qubit interaction. Two qubits can be included in a superconducting circuit, such that the spin-dependent supercurrents flowing through the two qubits interact magnetically. The interaction does not modify the polarization pseudovectors. As a result, the qubit-qubit interaction is of the Ising type,

$$
H=H_{1} \sigma_{1}^{z}+H_{2} \sigma_{2}^{z}+H_{12} \sigma_{1}^{z} \sigma_{2}^{z},
$$

choosing the $z$ axis along $\overrightarrow{\boldsymbol{\epsilon}}_{\mathrm{so}}$ for each qubit. The Ising-type interaction is sufficient to perform the CNOT operation, which, in combination with single-qubit operations, enables universal quantum computations. ${ }^{34}$

The CNOT gate is an operation on two qubits which has the effect of changing the state of one (target) qubit, only when the other (control) qubit is in the excited state. We propose a realization of CNOT gate using nonoscillating pulses of $H_{12} / \hbar$ of length $\tau$. The pulse shifts the relative phase between two qubit states, as follows: states $|\uparrow \uparrow\rangle$ and $|\downarrow \downarrow\rangle$ gain phase factor $\exp \left(i H_{12} \tau / \hbar\right)$ while states $|\uparrow \downarrow\rangle$ and $|\downarrow \uparrow\rangle$ gain phase factor $\exp \left(-i H_{12} \tau / \hbar\right)$. Tuning the length of the pulse such that $H_{12} \tau / \hbar=\pi / 4$, one obtains a phase-shift gate $G_{\text {phase }}$ that can be combined with Bloch sphere rotations by $\pi / 2$ of the control and target qubit around the coordinate axes $R_{x, y, z}(\pi / 2)$, to achieve the controlled NOT gate,

$$
\mathrm{CNOT}=e^{i \pi / 4} R_{z}^{(1)}(\pi / 2) R_{z}^{(2)}(-\pi / 2) R_{x}^{(2)}(\pi / 2) G_{\text {phase }} R_{y}^{(2)}(\pi / 2),
$$

where the superscripts (1) and (2) imply that the operation is performed on the control and target qubit, respectively. 
We have the opportunity to tune the interaction and also to switch it on and off. To give an example, consider the following circuit: two qubits with their superconducting loops connected in parallel [Fig. 3(b)]. The interaction energy can be tuned by the following parameters: the magnetic flux flowing through the superconducting loops $\left(\phi_{\text {loop1 }}\right.$ and $\left.\phi_{\text {loop2 }}\right)$ and the Josephson energy of the conventional Josephson junction $E_{j 3}$. To lowest order in the polarization pseudovectors $\left|\vec{\epsilon}_{\mathrm{so1}, 2}\right|$, the interaction takes the form,

$$
H_{12}=-\frac{\left|\vec{\epsilon}_{\mathrm{so} 1}\right|\left|\vec{\epsilon}_{\mathrm{so} 2}\right|}{|\widetilde{E}|} \cos \left(\varphi_{\tilde{E}}+\varphi_{1}\right) \cos \left(\varphi_{\tilde{E}}+\varphi_{2}\right),
$$

where the complex-valued energy $\widetilde{E}$ denotes $\widetilde{E}=E_{j 1} e^{i \varphi_{1}}$ $+E_{j 2} e^{i \varphi_{2}}+E_{j 3}$, and $\varphi_{\tilde{E}}$ denotes its complex argument $\varphi_{\tilde{E}}$ $=\tan ^{-1}[\operatorname{Im} \widetilde{E} / \operatorname{Re} \widetilde{E}]$. The angles $\varphi_{1,2}$ are proportional to the magnetic fluxes $\varphi_{1,2}=2 \pi\left(\phi_{\text {loop } 1,2} / \phi_{0}\right)$, with $\phi_{0}$ the magnetic flux quantum. Setting $\varphi_{1}=\varphi_{2}=\pi / 2$, it is possible to tune the interaction simply by controlling $E_{j 3}$. For $E_{j 3} \gg\left(E_{j 1}+E_{j 2}\right)$, the interaction is turned off $H_{12} \rightarrow 0$ while in the opposite limit $E_{j 3} \ll\left(E_{j 1}+E_{j 2}\right)$, the interaction achieves a maximum $H_{12}=-\left|\vec{\epsilon}_{\mathrm{so} 1}\right|\left|\vec{\epsilon}_{\mathrm{so} 2}\right| /|\widetilde{E}|$. Such control of the conventional Josephson energy can be achieved using exclusively electrical means, as was demonstrated in Ref. 29.

\section{E. Relaxation and decoherence}

Successful manipulation of the qubit quantum states can only be achieved at time scales shorter than the coherence time. This motivates us to discuss the mechanisms of relaxation and decoherence.

In the case of superconducting qubits, the main mechanisms leading to loss of coherence are well known. Coherence is lost due to coupling of the qubit dynamics to noises in magnetic flux and in the gate-induced charge of the Josephson junction. ${ }^{35}$ The same noises appear to be relevant for spin superconducting qubits. In this case, small deviations of flux modify the phase difference over the qubit junction while deviations of the gate voltage modify the modulus and direction of the polarization vector $\vec{\epsilon}_{\text {so. }}$. The phase difference and the modulus of $\vec{\epsilon}_{\mathrm{so}}$ are directly related to the qubit-level spacing $\Delta E=2\left|\vec{\epsilon}_{\mathrm{so}}\right||\sin (\varphi)|$. Their deviations lead to decoherence. The direction of $\vec{\epsilon}_{\text {so }}$ determines the orientation of the electron spin. Its deviation mixes the spin-qubit states, leading to energy relaxation.

We describe the effect of small deviations of flux $\delta \phi(t)$ and gate voltage $\delta V_{g}(t)$ by the following Hamiltonian:

$$
\begin{gathered}
H=\left[\left|\vec{\epsilon}_{\mathrm{so}}\right| \sin (\varphi)+\epsilon_{\|}\right] \sigma_{z}+\epsilon_{\perp} \sigma_{x}, \\
\epsilon_{\|}=\left|\vec{\epsilon}_{\mathrm{so}}\right| \cos (\varphi)\left(2 \pi / \phi_{0}\right) \delta \phi(t)+\epsilon_{\|}^{\prime} \sin (\varphi) e \delta V_{g}(t), \\
\epsilon_{\perp}=\epsilon_{\perp}^{\prime} \sin (\varphi) e \delta V_{g}(t),
\end{gathered}
$$

where $\epsilon^{\prime}$ terms describe the coupling of $\vec{\epsilon}_{\text {so }}$ to gate voltage, as follows: $\epsilon_{\|}^{\prime}=\left|\frac{d \vec{\epsilon}_{\mathrm{so}}}{d\left(e V_{g}\right)} \cdot \frac{\vec{\epsilon}_{\mathrm{so}}}{\mid \vec{\epsilon}_{\mathrm{so}}}\right|$ and $\epsilon_{\perp}^{\prime}=\left|\frac{d \vec{\epsilon}_{\mathrm{so}}}{d\left(e V_{o}\right)} \times \frac{\vec{\epsilon}_{\mathrm{so}}}{\left|\vec{\epsilon}_{\mathrm{so}}\right|}\right|$.

We have separated the coupling to the voltage and flux fluctuations into diagonal $\epsilon_{\|}$, and off-diagonal $\epsilon_{\perp}$ matrix elements. Therefore $\epsilon_{\|}$and $\epsilon_{\perp}$ are responsible for decoherence and relaxation, respectively. Since flux modulations do not modify the direction of $\vec{\epsilon}_{\mathrm{so}}$, they contribute only to the diagonal component $\epsilon_{\|}$. In contrast, voltage modulations contribute both to $\epsilon_{\|}$and $\epsilon_{\perp}$.

We estimate the frequency-dependent noises using the fluctuation-dissipation theorem. Not all types of noises can be estimated in this way. For instance, $1 / f$ noise of flux may present a problem. Nevertheless, these noises can be quantified experimentally. ${ }^{35}$ For illustration of the use of the fluctuation-dissipation theorem, we refer to the circuit in Fig. 3(a) $\left(E_{j 1} \gg E_{j 2}\right)$. However, the following discussion is not restricted to this specific circuit.

The flux response function to external driving is determined by modeling the qubit as a parallel $R L$ circuit. $L$ denotes the Josephson inductance of the circuit. In the case of Fig. 3(a), with $E_{j 1} \gg E_{j 2}$ and $L=\left(\phi_{0} / 2 \pi\right)\left[\hbar / 2 e E_{j 1} \cos (\varphi)\right] . R$ describes energy dissipation to the environment. One usually assumes $R$ to be in the same order of magnitude as the vacuum impedance $Z_{0}=\mu_{0} c \approx 377 \Omega$, describing loss of energy by radiation.

The gate-voltage response function is determined by modeling the qubit tunnel junction as an $R C$ circuit connected in series. Here, $C$ is not the capacitance formed between the gate electrode and the quantum dot. We recognize that the response of gate voltage is dominated by the much larger capacitance formed between the gate electrode and the superconducting leads. The typical charging time associated to this capacitance is long compared to the time scale of the qubit dynamics, $R C \gg \hbar /\left|\vec{\epsilon}_{\mathrm{so}}\right|, \hbar / k_{B} T$.

We make the following assumptions regarding the time scale of relaxation and decoherence. The relaxation time $\tau_{R}$ is typically much longer than the time scale of the qubit dynamics $\tau_{R} \gg \hbar /\left|\vec{\epsilon}_{\mathrm{so}}\right|, \hbar / k_{B} T$. The decoherence time can be shorter $\tau_{D}<\tau_{R}$ but is commonly assumed to be much longer than $\hbar / k_{B} T$. This sets the relevant frequency of noise $\omega$ $<k_{B} T / \hbar$, i.e., low-frequency classical noise.

We estimate the magnetic-flux noise,

$$
S_{\phi}=\left(\frac{\phi_{0}}{2 \pi}\right)^{2} \frac{1}{8 \pi R G_{Q}}\left(\frac{\hbar}{E_{j 1} \cos (\varphi)}\right)^{2} \frac{k_{B} T}{\hbar},
$$

where $G_{Q}=e^{2} /(\pi \hbar) \approx 7.75 \times 10^{-5} \Omega^{-1}$ is the quantum of conductance.

The low-frequency gate voltage noise is given by

$$
S_{V_{g}}=2 R k_{B} T \text {. }
$$

Since we have neglected charging effects related to the large capacitance $C$, the remaining contribution to gate-voltage noise is the Ohmic response of the resistance $R$.

We estimate the decoherence time $\tau_{D}$ using the following expression:

$$
\begin{gathered}
\frac{1}{\tau_{D}}=\left(\frac{1}{\tau_{D}}\right)_{\phi}+\left(\frac{1}{\tau_{D}}\right)_{V_{g}}, \\
\left(\frac{1}{\tau_{D}}\right)_{\phi}=\frac{2}{\hbar^{2}}\left[\left|\vec{\epsilon}_{\mathrm{so}}\right| \cos (\varphi)\right]^{2} S_{\phi}=\frac{1}{4 \pi R G_{Q}}\left(\frac{\left|\vec{\epsilon}_{\mathrm{so}}\right|}{E_{j 1}}\right)^{2} \frac{k_{B} T}{\hbar},
\end{gathered}
$$




$$
\left(\frac{1}{\tau_{D}}\right)_{V_{g}}=\frac{e^{2}}{2 \hbar^{2}}\left(\epsilon_{\|}^{\prime}\right)^{2} \sin ^{2}(\varphi) S_{V_{g}}=\pi R G_{Q}\left(\epsilon_{\|}^{\prime}\right)^{2} \sin ^{2}(\varphi) \frac{k_{B} T}{\hbar} .
$$

There are two contributions to decoherence: the first $\left(1 / \tau_{D}\right)_{\phi}$, describes decoherence due to coupling to flux noise. The second $\left(1 / \tau_{D}\right)_{V_{g}}$, describes decoherence due to coupling to gate-voltage noise.

Let us focus on the coupling to flux noise. We recall our initial assumption $\tau_{D}^{-1} \ll k_{B} T / \hbar, \Delta E / \hbar$. This sets a lower bound on $E_{j 1}: E_{j 1}^{2} \gg\left|\vec{\epsilon}_{\mathrm{so}}\right|^{2} / 4 \pi R G_{Q}$. The physical interpretation of this condition is as follows: the qubit junction must be shunted by a circuit element with sufficiently low inductance (high Josephson energy) to reduce sensitivity to flux noise. Otherwise, the high sensitivity to flux noise would prevent any coherent manipulation of the qubit state. The same requirement holds for other superconducting qubits, ${ }^{35}$ which are designed to fulfill it. In this case, the sensitivity to magnetic-flux noise scales as $\left(E_{j 2} / E_{j 1}\right)^{2}$. Superconducting spin qubits are significantly less sensitive to such noise. For the superconducting spin qubit, the scaling factor is $\left(\left|\vec{\epsilon}_{\mathrm{so}}\right| / E_{j 1}\right)^{2}$, smaller by a factor of the spin-orbit coupling strength squared. Therefore, we predict significantly longer coherence times for the superconducting spin qubit compared to other superconducting qubits. The lower sensitivity to noise is a compensation for the relatively smaller readout currents.

We mention that the expressions in Eqs. (10) and (11) are valid only for $\tau_{D}^{-1} \ll R / L$. This sets an upper bound on the temperature in terms of the resistance $R$ and $E_{j 1}$ : $R G_{Q} E_{j 1}\left(E_{j 1} /\left|\vec{\epsilon}_{\mathrm{so}}\right|\right) \gg k_{B} T$. The condition is easily achieved in the typical temperature regime required to induce superconductivity.

Let us turn our attention to the coupling to gate-voltage noise. The sensitivity to voltage fluctuations is described by $\epsilon_{\|}^{\prime}$. As we show in Sec. IV, this parameter depends strongly on gate voltage and on the microscopic parameters describing the qubit junction. It can be estimated to be $\epsilon_{\|}^{\prime}$ $\approx\left|\vec{\epsilon}_{\mathrm{so}}\right| / E_{C} \ll 1$, where $E_{C}$ is the quantum dot charging energy.

We can compare the coupling to flux noise with the coupling to gate-voltage noise. The flux noise contribution dominates provided $E_{j 1}<E_{C} / R G_{Q}$. The energy scale $E_{C} / R G_{Q}$ is typically very high so that the condition $E_{j 1}$ $<E_{C} / R G_{Q}$ is satisfied. Therefore, we conclude that in typical devices coupling to flux noise will be the dominating decoherence mechanism. If $E_{j 1}$ is large enough such that the opposite limit holds $E_{j 1}>E_{C} / R G_{Q}$, the coupling to gatevoltage noise dominates and decoherence times are longer.

Let us compare the inverse decoherence time with the Rabi frequency. We can estimate the Rabi frequency to be on the order of $\Omega_{R} \approx\left|\vec{\epsilon}_{\mathrm{so}}\right| / \hbar$, for sufficiently high driving amplitudes. The decoherence time can be estimated as $\tau_{D}^{-1}$ $\approx\left(\left|\vec{\epsilon}_{\mathrm{so}}\right| k_{B} T / 4 \pi R G_{Q} E_{j 1}^{2}\right) \Omega_{R}$. We see that the number of coherent Rabi oscillations that can be realized $\Omega_{R} / \tau_{D}^{-1}$ scales as $E_{j 1} /\left|\vec{\epsilon}_{\mathrm{so}}\right|$. This can be compared to the case of superconducting qubits, where $\Omega_{R} / \tau_{D}^{-1}$ scales as $E_{j 2} / E_{j 1}$, i.e., smaller by a factor of the spin-orbit coupling strength. This is a consequence of the lower sensitivity to flux noise of the supercon- ducting spin qubit. In conclusion, we predict that superconducting spin qubits have longer coherence times compared to other superconducting qubits, an important advantage in view of performing a large number of coherent quantum operations required by quantum computation schemes.

Further, let us focus on the relaxation time $\tau_{R}$. In the limit $\Delta E<k_{B} T, \tau_{R}$ can be estimated as follows:

$$
\frac{1}{\tau_{R}}=\frac{e^{2}}{\hbar^{2}}\left(\epsilon_{\perp}^{\prime}\right)^{2} \sin ^{2}(\varphi) S_{V_{g}}=\pi R G_{Q}\left(\epsilon_{\perp}^{\prime}\right)^{2} \sin ^{2}(\varphi) \frac{k_{B} T}{\hbar} .
$$

In the opposite limit $\Delta E>k_{B} T$, the relaxation time becomes

$$
\frac{1}{\tau_{R}}=\pi R G_{Q}\left(\epsilon_{\perp}^{\prime}\right)^{2} \sin ^{2}(\varphi) \frac{\Delta E}{\hbar} .
$$

We can compare the relaxation and decoherence times. The typical case is when decoherence is dominated by flux noise, i.e., $E_{j 1}<E_{C} / R G_{Q}$. In this case, the decoherence time is always much shorter than the relaxation time. In the opposite case, when $E_{j 1}>E_{C} / R G_{Q}$, we find that $\tau_{D} / \tau_{R}=\left(\epsilon_{\perp}^{\prime} / \epsilon_{\|}^{\prime}\right)^{2}$. The ratio $\epsilon_{\perp}^{\prime} / \epsilon_{\|}^{\prime}$ depends on microscopic junction parameters and is discussed in Sec. IV. Typically, $\epsilon_{\perp}^{\prime} / \epsilon_{\|}^{\prime} \approx \Delta / E_{C} \leq 1$. In case $\tau_{D} \approx \tau_{R}$, the decoherence rate is determined by the relaxation time.

\section{MICROSCOPIC DESCRIPTION}

\section{A. Hamiltonian}

The total Hamiltonian comprises terms describing leads (left and right), quantum dot and tunneling between electronic states in the leads and in the dot,

$$
\begin{gathered}
\hat{H}=\hat{H}_{L}+\hat{H}_{R}+\hat{H}_{\mathrm{QD}}+\hat{H}_{T} \\
=\hat{H}_{0}+\hat{H}_{T} .
\end{gathered}
$$

The electronic states of the left lead are labeled by $l$ and spin index $\sigma$ and are affected by the superconducting order parameter $\Delta e^{i \varphi_{L}}$,

$$
\hat{H}_{L}=\sum_{l, \sigma} \xi_{l} a_{l \sigma}^{\dagger} a_{l \sigma}+\sum_{\sigma^{\prime}} \Delta e^{i \varphi_{L}} g^{\sigma \sigma^{\prime}} a_{l \sigma^{\prime}}^{\dagger} a_{l \sigma}^{\dagger}+g^{\sigma \sigma^{\prime}} \Delta e^{-i \varphi_{L}} a_{l \sigma} a_{l \sigma^{\prime}},
$$

where $a^{\dagger}(a)$ denotes the creation (annihilation) operators and $\xi$ denotes the energy of the levels in the normal-metal state, counted from the chemical potential of the lead. In the superconducting state, the energy of the levels is $\epsilon_{l}=\sqrt{\xi_{l}^{2}+\Delta^{2}}$.

The terms of $\hat{H}_{L}$ that depend on $\Delta e^{i \varphi_{L}}$ couple timereversed states. To underline this, we mention that the electronic states labeled by index $l$ are not momentum eigenstates but rather the boxlike single-particle levels (in normal state) that are superpositions of plane waves with opposite momenta. The time-reversal operator does not change index $l$. Time-reversal changes the sign of the spin. We take this into account by using the antisymmetric matrix $g=i \sigma^{2}$. This ensures that the two electrons forming a Cooper pair in the leads are a spin singlet. Similarly, electronic states in the right lead are labeled by $r$ and are affected by $\Delta e^{i \varphi_{R}}$. 
The Hamiltonian of the quantum dot contains charging energy term along with the terms describing noninteracting electrons in levels labeled by $m, \Sigma_{m} \xi_{m} a_{m}^{\dagger} a_{m}$,

$$
\hat{H}_{\mathrm{QD}}=\sum_{m} \xi_{m} a_{m}^{\dagger} a_{m}+\left(\hat{N}-N_{0}\right)^{2} E_{C}
$$

with $\hat{N}$ being the operator of the total number of electrons $\hat{N}=\Sigma_{m} a_{m}^{\dagger} a_{m} . N_{0}=V_{g} C_{g} / e$ represents the effect of gate voltage which allows to tune the total number of electrons in the dot. $C_{g}$ here is the capacitance to the gate. Since it only appears in combination with gate voltage, we find it convenient to rescale the gate voltage $V_{g} C_{g} / C \rightarrow V_{g}$, with $C$ being the total capacitance of the junction. In these units, $N_{0}=e V_{g} / E_{C}$.

We stress that all these terms are not affected by spin-orbit coupling. The reason for this is that spin-orbit coupling retains the double degeneracy of the electronic states. Instead of spin doublets, they become Kramers doublets so index $\sigma$ in this case refers to the components of Kramers doublet, rather than to the original spin state. The only place where spin-orbit coupling plays a role is the tunneling part of the Hamiltonian and can be included into spin-dependent tunneling amplitudes, ${ }^{33}$

$$
\hat{H}_{T}=\sum_{l, r, m, \rho, \sigma} T_{l m}^{\rho \sigma} a_{m \sigma}^{\dagger} a_{l \rho}+T_{m r}^{\rho \sigma} a_{r \sigma}^{\dagger} a_{m \rho}+\text { H.c. }
$$

where $\rho$ and $\sigma$ are spin indexes.

As a result of time-reversal symmetry, we may write the tunneling matrices in spin space,

$$
T_{l m}^{\rho \sigma}=T_{l m}^{0} \delta_{\rho \sigma}+i \sum_{j=1}^{3} t_{l m}^{j} \sigma_{\rho \sigma}^{j},
$$

where $T_{l m}^{0}$ and $t_{l m}^{j}$ are chosen to be real. The spinindependent part is symmetric $T_{l m}^{0}=T_{m l}^{0}$ and spin-dependent part is antisymmetric $t_{l m}^{j}=-t_{m l}^{j}$ with respect to interchanging the state indices.

In principle, the tunneling matrix elements can be evaluated by computing overlap of spin-dependent wave functions of corresponding components of Kramers doublets. It is known that wave functions become random either because of scattering at defects in the leads and in the dot or because of chaoticity of classical electron motion in the dot. If we consider extended system, the statistics of spin-dependent part of such overlaps is governed by length scale $l_{\text {sf }}$ that is a spin-flip length, induced by spin-orbit scattering. At distances exceeding this scale, spin orientation of Kramers doublets becomes completely random so that $T^{0}$ is on the order of $|\vec{t}|$. The length scale $l_{\text {sf }}$ corresponds to time scale $\tau_{\text {sf }}$, spin-flip time. If we apply this now to a quantum dot, the spin structure becomes random for each level in the dot, provided the meanlevel spacing in the dot $\delta_{S}$ is comparable or smaller than $\hbar / \tau_{\text {sf. }}$. The ratio of the two defines the strength of spin-orbit coupling. For our estimations, we assume $\vec{t} / T^{0} \approx 0.1$ and random distribution of the direction. Preliminary experiments with InAs nanowires ${ }^{36}$ confirm this by order of magnitude.

\section{B. Josephson energy}

In the following, we provide a microscopic description of the Josephson effect in the presence of spin-orbit coupling. We treat Cooper-pair transport through a quantum dot in the regime of Coulomb blockade, with no bias voltage and disregarding effects of thermal excitation. Within the Coulomb diamonds, the only mechanism of transport is cotunneling. Four tunneling events are sufficient to transfer a Cooper pair between the leads ${ }^{37}$ and these processes dominate for sufficiently small normal-state conductances $G_{N} \ll G_{Q}$. We employ fourth-order perturbation theory in the tunneling amplitudes and find the Josephson energy as the perturbation correction to the ground-state energy.

The ground state of the unperturbed Hamiltonian $\hat{H}_{0}$ $=\hat{H}_{L}+\hat{H}_{R}+\hat{H}_{\mathrm{QD}}$ is degenerate with respect to the number of Cooper pairs on the superconducting leads. The wave function can be written as a direct product of three wave functions: $\psi\left(N_{L}, N_{R}\right)=\psi_{L}\left(N_{L}\right) \otimes \psi_{\mathrm{QD}} \otimes \psi_{R}\left(N_{L}\right)$ corresponding to the two leads and the quantum dot. $N_{L}$ and $N_{R}$ represent the number of electrons in the respective leads.

In the presence of tunneling, the degeneracy of the ground state is lifted and the energy splitting between states with different number of Cooper pairs in the leads corresponds to the Josephson energy,

$$
\begin{aligned}
E_{J}(\varphi)= & \left\langle\psi(-2,2)\left|\hat{T}^{(4)}\right| \psi(0,0)\right\rangle e^{i \varphi} \\
& +\left\langle\psi(2,-2)\left|\hat{T}^{(4)}\right| \psi(0,0)\right\rangle e^{-i \varphi} \\
= & 2 \operatorname{Re}\left\{\left\langle\psi(-2,2)\left|\hat{H}_{T}^{(4)}\right| \psi(0,0)\right\rangle e^{i \varphi}\right\},
\end{aligned}
$$

where $N_{L}$ and $N_{R}$ have been set to zero.

Here, operator $\hat{T}^{(4)}$ represents the correction to the amplitude of the fourth order in tunneling Hamiltonian,

$$
\hat{T}^{(4)}=\hat{H}_{T} \frac{1}{E_{0}-\hat{H}_{0}} \hat{H}_{T} \frac{1}{E_{0}-\hat{H}_{0}} \hat{H}_{T} \frac{1}{E_{0}-\hat{H}_{0}} \hat{H}_{T} .
$$

The structure of the fourth-order correction is as follows. Each tunneling operator $\hat{H}_{T}$ describes the transition of one electron between a state in the dot and a state in one of the superconducting leads. There are 24 distinct sequences resulting from the permutations of the four tunneling events considered. Each process involves three intermediary states. The energies of the virtual states appear as the three denominators of Eq. (20). The three energy denominators unambiguously characterizes the sequence of tunneling events.

In contrast, the spin structure does not depend on the order of individual tunneling events. The spin structure of $\hat{T}^{(4)}$ can be recovered from the product of four tunneling amplitudes describing hopping of the two electrons between the leads and the quantum dot,

$$
\begin{aligned}
\sum_{l, r} g^{T} T_{l m}^{T} g T_{l n} T_{n r} g^{T} T_{m r}^{T} g \delta\left(\xi_{L}-\xi_{l}\right) \delta\left(\xi_{R}-\xi_{r}\right) \\
=\sum_{l, r} T_{m l} T_{l n} T_{n r} T_{r m} \delta\left(\xi_{L}-\xi_{l}\right) \delta\left(\xi_{R}-\xi_{r}\right)=P_{m n}^{0}\left(\xi_{L}, \xi_{R}\right) \\
\quad+i \vec{P}_{m n}\left(\xi_{L}, \xi_{R}\right) \cdot \vec{\sigma}
\end{aligned}
$$


where $m$ and $n$ label states of the quantum dot.

Let us explain the properties of the two terms in Eq. (21). In the following, we neglect terms proportional to the square of the small term $\vec{t} / T^{0}$. Since the leads have continuous spectra, it is convenient to introduce transport rates which are continuous functions of energy, $\Gamma_{L(R), m}(\xi)$ $=2 \pi / \hbar \Sigma_{l(r)}\left|T_{m l(r)}^{0}\right|^{2} \delta\left(\xi-\xi_{l(r)}\right)$. The spin-independent term $P_{m n}^{0}\left(\xi_{L}, \xi_{R}\right)$, has different properties in the case $m=n$, compared to the case $m \neq n$. Terms diagonal in the dot levels have the following simple form: $P_{m m}^{0}\left(\xi_{L}, \xi_{R}\right)$ $=(\hbar / 2 \pi)^{2} \Gamma_{L, m}\left(\xi_{L}\right) \Gamma_{R, m}\left(\xi_{R}\right)$. As a result, they are always positive. In contrast, the sign of $P_{m n}^{0}\left(\xi_{L}, \xi_{R}\right)$ when $m \neq n$ can be related to the parities of the wave functions of the two dot states. In analogy to the case of a particle in a box, the parity of wave functions in a quantum dot alternates between neighboring states. As a result, the sign of $P_{m, n \neq m}^{0}\left(\xi_{L}, \xi_{R}\right)$ alternates and the sum over all states in the quantum dot averages out, $\Sigma_{m, n \neq m} P_{m n}^{0}\left(\xi_{L}, \xi_{R}\right) \approx 0$.

The contribution corresponding to the spin-dependent term in Eq. (21) changes sign for different spin orientations. Therefore, this term cancels out if the levels $m$ and $n$ are both either empty or filled with electrons, as there is no asymmetry between spin-up and spin-down terms. However, if either state $m$ or $n$ is the state filled with a single electron, the contribution is finite, giving rise to the spin-polarization effect.

After calculating the energy denominators for each tunneling sequence and integrating over all states in the leads and all pairs of states in the dot, we obtain the following result for the spin-independent Josephson energy: ${ }^{38}$

$$
\begin{aligned}
E_{j}= & \frac{\Delta^{2}}{4} \int_{-\infty}^{\infty} \frac{d \xi_{L}}{\epsilon_{L}} \int_{-\infty}^{\infty} \frac{d \xi_{R}}{\epsilon_{R}}\left[\sum_{M_{1}, M_{2}} P_{M_{1} M_{2}}^{0}\left(\xi_{L}, \xi_{R}\right) J_{e e}\left(\xi_{L}, \xi_{R}, \xi_{M_{1}}, \xi_{M_{2}}\right)+\sum_{m_{1}, m_{2}} P_{m_{1} m_{2}}^{0}\left(\xi_{L}, \xi_{R}\right) J_{e e}\left(\xi_{L}, \xi_{R}, \xi_{m_{1}}, \xi_{m_{2}}\right)\right. \\
& -2 \sum_{M, m} P_{M m}^{0}\left(\xi_{L}, \xi_{R}\right) J_{e h}\left(\xi_{L}, \xi_{R}, \xi_{M}, \xi_{m}\right)+\sum_{M} P_{M 0}^{0}\left(\xi_{L}, \xi_{R}\right)\left(J_{e e}\left(\xi_{L}, \xi_{R}, \xi_{M}, \xi_{0}\right)-J_{e h}\left(\xi_{L}, \xi_{R}, \xi_{M}, \xi_{0}\right)\right)+\sum_{m} P_{0 m}^{0}\left(\xi_{L}, \xi_{R}\right) \\
& \left.\times\left(J_{h h}\left(\xi_{L}, \xi_{R}, \xi_{0}, \xi_{m}\right)-J_{e h}\left(\xi_{L}, \xi_{R}, \xi_{0}, \xi_{m}\right)\right)-2 P_{00}^{0}\left(\xi_{L}, \xi_{R}\right) J_{e h}\left(\xi_{L}, \xi_{R}, \xi_{0}, \xi_{0}\right)\right]
\end{aligned}
$$

and for the polarization pseudovector,

$$
\begin{aligned}
\vec{\epsilon}_{\mathrm{so}}= & \frac{\Delta^{2}}{4} \int_{-\infty}^{\infty} \frac{d \xi_{L}}{\epsilon_{L}} \int_{-\infty}^{\infty} \frac{d \xi_{R}}{\epsilon_{R}}\left[\sum_{M} \vec{P}_{M 0}\left(\xi_{L}, \xi_{R}\right)\left(J_{e e}\left(\xi_{L}, \xi_{R}, \xi_{M}, \xi_{0}\right)+J_{e h}\left(\xi_{L}, \xi_{R}, \xi_{M}, \xi_{0}\right)\right)-\sum_{m} \vec{P}_{0 m}\left(\xi_{L}, \xi_{R}\right)\left(J_{h h}\left(\xi_{L}, \xi_{R}, \xi_{0}, \xi_{m}\right)\right.\right. \\
& \left.\left.+J_{e h}\left(\xi_{L}, \xi_{R}, \xi_{0}, \xi_{m}\right)\right)\right],
\end{aligned}
$$

where $m, m_{1}$, and $m_{2}$ label filled dot states, $M, M_{1}$, and $M_{2}$ empty dot states and 0 labels the half-filled state. We have used the notation $\epsilon_{L, R}=\sqrt{\Delta^{2}+\xi_{L, R}^{2}}$. The $J$ functions contain the energy denominators and are different for processes where the Cooper pair is transferred via two electrons, two holes or one electron, and one hole, respectively:

$$
\begin{aligned}
J_{e e}\left(\xi_{L}, \xi_{R}, \xi_{m}, \xi_{n}\right)= & \frac{1}{\epsilon_{L}+\xi_{m}+E(e)} \frac{1}{\epsilon_{L}+\epsilon_{R}} \frac{1}{\epsilon_{R}+\xi_{n}+E(e)}+\frac{1}{\epsilon_{L}+\xi_{n}+E(e)} \frac{1}{\epsilon_{L}+\epsilon_{R}} \frac{1}{\epsilon_{R}+\xi_{m}+E(e)} \\
& +\frac{1}{\epsilon_{L}+\xi_{m}+E(e)} \frac{1}{\xi_{m}+\xi_{n}+E(2 e)} \frac{1}{\epsilon_{R}+\xi_{m}+E(e)}+\frac{1}{\epsilon_{L}+\xi_{n}+E(e)} \frac{1}{\xi_{m}+\xi_{n}+E(2 e)} \frac{1}{\epsilon_{R}+\xi_{m}+E(e)} \\
& +\frac{1}{\epsilon_{L}+\xi_{m}+E(e)} \frac{1}{\xi_{m}+\xi_{n}+E(2 e)} \frac{1}{\epsilon_{R}+\xi_{n}+E(e)}+\frac{1}{\epsilon_{L}+\xi_{n}+E(e)} \frac{1}{\xi_{m}+\xi_{n}+E(2 e)} \frac{1}{\epsilon_{R}+\xi_{n}+E(e)}, \\
J_{h h}\left(\xi_{L}, \xi_{R}, \xi_{m}, \xi_{n}\right)= & \frac{1}{\epsilon_{L}-\xi_{m}+E(-e)} \frac{1}{\epsilon_{L}+\epsilon_{R}} \frac{1}{\epsilon_{R}-\xi_{n}+E(-e)}+\frac{1}{\epsilon_{L}-\xi_{n}+E(-e)} \frac{1}{\epsilon_{L}+\epsilon_{R}} \frac{1}{\epsilon_{R}-\xi_{m}+E(-e)} \\
& +\frac{1}{\epsilon_{L}-\xi_{m}+E(-e)} \frac{1}{-\xi_{m}-\xi_{n}+E(-2 e)} \frac{1}{\epsilon_{R}-\xi_{m}+E(-e)} \\
& +\frac{1}{\epsilon_{L}-\xi_{n}+E(-e)} \frac{1}{-\xi_{m}-\xi_{n}+E(-2 e)} \frac{1}{\epsilon_{R}-\xi_{m}+E(-e)} \\
& +\frac{1}{\epsilon_{L}-\xi_{m}+E(-e)} \frac{1}{-\xi_{m}-\xi_{n}+E(-2 e)} \frac{1}{\epsilon_{R}-\xi_{n}+E(-e)} \\
& +\frac{1}{\epsilon_{L}-\xi_{n}+E(-e)} \frac{1}{-\xi_{m}-\xi_{n}+E(-2 e)} \frac{1}{\epsilon_{R}-\xi_{n}+E(-e)}
\end{aligned}
$$




$$
\begin{aligned}
J_{e h}\left(\xi_{L}, \xi_{R}, \xi_{m}, \xi_{n}\right)= & \frac{1}{\epsilon_{L}+\xi_{m}+E(e)} \frac{1}{\epsilon_{L}+\epsilon_{R}} \frac{1}{\epsilon_{L}-\xi_{n}+E(-e)}+\frac{1}{\epsilon_{R}+\xi_{m}+E(e)} \frac{1}{\epsilon_{L}+\epsilon_{R}} \frac{1}{\epsilon_{R}-\xi_{n}+E(-e)} \\
& +\frac{1}{\epsilon_{L}+\xi_{m}+E(e)} \frac{1}{\xi_{m}-\xi_{n}+\epsilon_{L}+\epsilon_{R}} \frac{1}{\epsilon_{R}+\xi_{m}+E(e)}+\frac{1}{\epsilon_{R}-\xi_{n}+E(-e)} \frac{1}{\xi_{m}-\xi_{n}+\epsilon_{L}+\epsilon_{R}} \frac{1}{\epsilon_{R}+\xi_{m}+E(e)} \\
& +\frac{1}{\epsilon_{L}+\xi_{m}+E(e)} \frac{1}{\xi_{m}-\xi_{n}+\epsilon_{L}+\epsilon_{R}} \frac{1}{\epsilon_{L}-\xi_{n}+E(-e)}+\frac{1}{\epsilon_{R}-\xi_{n}+E(-e)} \frac{1}{\xi_{m}-\xi_{n}+\epsilon_{L}+\epsilon_{R}} \frac{1}{\epsilon_{L}-\xi_{n}+E(-e)}
\end{aligned}
$$

The above expressions can be used to calculate the spinindependent and spin-dependent parts of the Josephson energy. However, they require knowledge of all the energy levels and corresponding tunneling amplitudes of the quantum dot. Here, we do not intend to develop a model of the quantum dot and extract these parameters. Such a model would have to account for the unavoidable defects present in a typical experimental device. Our intention is to use the above expressions as a useful starting point for estimations and for distinguishing different parameter regimes.

Instead of using analytic or numerical calculations, the polarization vector can be determined experimentally using spectroscopic techniques typical for superconducting qubits. ${ }^{24}$ From the absorption spectra of the qubit junction, one determines the spacing between the qubit states $\Delta E$ $=2\left|\vec{\epsilon}_{\mathrm{so}}\right||\sin (\varphi)|$, at a fixed superconducting phase difference. Therefore, one can find the modulus of the polarization vector.

We do not suggest a method to determine the orientation in three-dimensional space of the polarization axis in an experiment. The precise orientation is of no relevance here. What is important is that the orientation of this axis can be modified by changing the gate voltage or by applying external magnetic fields. In Sec. II, we have related rotations of the polarization axis to manipulation of the qubit state.

\section{ESTIMATIONS}

Let us estimate the typical magnitude of $\vec{\epsilon}_{\text {so }}$ and compare it to the magnitude of spin-independent Josephson energy. We will see that the relative magnitudes as well as its absolute value cannot be just estimated by the typical strength of spin-orbit coupling $|\vec{t}| / T_{0}$. The estimations depend on three energy scales in the problem: $\delta_{S}, E_{C}$, and $\Delta$. Besides, it depends on the energy distance to the diamond edge.

It has been already shown in Ref. 37 that Josephson energy and critical current exhibit spectacular peculiarities at the diamond edge. These peculiarities are no singularities. This is related to the diamond structure in the presence of superconductivity. ${ }^{37}$ There is a bistability region in interval of width $\Delta\left(e V_{g}\right)=2 \Delta$ around each diamond edge. The singularities in denominators of perturbation theory occur at the edges of bistability region for the charged state of higher energy. We always assume the dot to be in the lowest-energy state. This saves us from singularities. However, the proximity to the point of singularity gives a spectacular increase or decrease in Josephson energy at the diamond edge. To ac- count for this in our estimations, we introduce the minimal energy distance to the edge of Coulomb diamond $E_{ \pm}$ $=|\min [E(e), E(-e)]|>\Delta$. We envisage two separate situations: $E_{ \pm} \approx E_{C}$ and $E_{ \pm} \ll E_{C}$.

The dominant contribution to the Josephson energy consists of the terms with smallest energy denominators. The magnitude of the energy denominators depends on the energy of the dot levels involved in tunneling. We find that the energy interval for quantum-dot levels involved in the dominating contribution has the width $E_{ \pm}$, even in the regime $E_{ \pm} \gg \Delta$. Let us define the number of levels within this interval $N_{S}$ as the integer part of $E_{ \pm} / \delta_{S}+1$. If $\delta_{S} \geq E_{ \pm}$, the Josephson energy is dominated by the contribution of a single level. If $\delta_{S}<E_{ \pm}$, there are multiple levels participating in the dominating contribution.

Using Eq. (22), we find the following estimate for the spin-independent Josephson energy:

$$
E_{j} \approx N_{S} \frac{\Gamma_{L} \Gamma_{R}}{\Delta} \frac{\Delta^{2}}{E_{ \pm}^{2}} .
$$

Similarly, we can estimate the spin-dependent component using Eq. (23),

$$
\left|\vec{\epsilon}_{\mathrm{so}}\right| \approx \sqrt{N_{S}} \frac{\Gamma_{L} \Gamma_{R}}{\Delta} \frac{\Delta^{2}}{E_{ \pm}\left[\max \left(E_{ \pm}, \delta_{S}\right)\right]} \frac{|\vec{t}|}{T_{0}} .
$$

In all parameter regimes considered, both $E_{j}$ as well as $\left|\vec{\epsilon}_{\mathrm{so}}\right|$ increase as $E_{ \pm} / \Delta$ decreases, i.e., as we approach the edges of the Coulomb diamond. We can explain this in terms of the energy of the intermediary virtual states. In the middle of the Coulomb diamond, the energy cost of adding an electron to the quantum dot is maximum and the high energy of the intermediary states reduces the probability of Cooperpair tunneling. Toward the edges of the diamond, tunneling processes involving the state closest to resonance will have intermediary states that are lower in energy, resulting in the increase in Josephson coupling.

Let us turn our attention to the multilevel regime, where Josephson tunneling is a result of the interference of tunneling processes that involve all pairs of the $N_{S}$ relevant states. The spin-dependent and spin-independent terms do not scale in the same way with the number of levels involved. In the case of $E_{j}$, the dominant contribution results from summation of diagonal spin-independent elements $P_{m m}^{0}\left(\xi_{L}, \xi_{R}\right)$. As a result, $E_{j}$ scales with the number of levels $N_{S}$. In the case of $\vec{\epsilon}_{\text {so }}$, the result of summation over tunneling contributions is equivalent to the distance traveled in a random walk in three 
dimensions, after $N_{S}$ steps. The average result in this case scales as $\sqrt{N_{S}}$.

The ratio of spin-dependent to spin-independent contributions is

$$
\frac{\left|\vec{\epsilon}_{\mathrm{so}}\right|}{E_{j}} \approx \frac{1}{\sqrt{N_{S}}} \frac{E_{ \pm}}{\max \left(E_{ \pm}, \delta_{S}\right)} \frac{|\vec{t}|}{T_{0}} .
$$

Apart from the trivial dependence on spin-orbit coupling strength, the ratio $\left|\vec{\epsilon}_{\mathrm{so}}\right| / E_{j}$ is further reduced by factors that depend on the gate voltage. We distinguish two important regimes: the single-level regime $\delta_{S} \geq E_{C}$, and the multilevel regime $\delta_{S} \ll E_{C}$, where multiple dot states contribute.

In the single-level regime, we find $\left|\vec{\epsilon}_{\mathrm{so}}\right| / E_{j} \approx E_{ \pm} / \delta_{S}$. Thus, it is large in the middle of the diamond, where it scales as $E_{C} / \delta_{S}$, decreasing toward the edge, where it scales as $\Delta / \delta_{S}$. The behavior has a simple explanation in terms of energy of intermediary virtual states. The dominant contribution to $E_{j}$ arises from processes involving tunneling of both Cooperpair electrons through the dot level closest to resonance. Such processes do not contribute to the polarization effect; as we have seen, only processes involving two different levels contribute. Thus, the energy of intermediary states decreases faster as we approach the diamond edge for dominant processes contributing to $E_{j}$.

We can also estimate the dependence of $\vec{\epsilon}_{\text {so }}$ on gate voltage. Let us define the change in $\vec{\epsilon}_{\text {so }}$ with gate voltage in the parallel and perpendicular directions, as we did in Sec. II: $\epsilon_{\|}^{\prime}=\left|\frac{d \vec{\epsilon}_{\mathrm{so}}}{d\left(e V_{g}\right)} \cdot \frac{\vec{\epsilon}_{\mathrm{so}}}{\left|\vec{\epsilon}_{\mathrm{so}}\right|}\right|$ and $\epsilon_{\perp}^{\prime}=\left|\frac{d \overrightarrow{\mathrm{s}}_{\mathrm{so}}}{d\left(e V_{g}\right)} \times \frac{\vec{\epsilon}_{\mathrm{so}}}{\left|\overrightarrow{\boldsymbol{\epsilon}}_{\mathrm{so}}\right|}\right|$. We have shown that the sensitivity of $\vec{\epsilon}_{\mathrm{so}}$ to gate voltage is an important mechanism of relaxation and decoherence. Furthermore, we have shown in Sec. II that change in $\overrightarrow{\boldsymbol{\epsilon}}_{\mathrm{so}}$ perpendicular to its initial direction is an essential ingredient for electrically driven Rabi oscillations.

We find the following estimates for the parallel and perpendicular derivatives with respect to $\vec{\epsilon}_{\mathrm{so}}$ :

$$
\begin{aligned}
& \left|\frac{d \overrightarrow{\boldsymbol{\epsilon}}_{\mathrm{so}}}{d\left(e V_{g}\right)} \cdot \frac{\overrightarrow{\boldsymbol{\epsilon}}_{\mathrm{so}}}{\left|\overrightarrow{\boldsymbol{\epsilon}}_{\mathrm{so}}\right|}\right| \approx \frac{\Gamma_{L} \Gamma_{R}}{\Delta} \frac{\Delta^{2}}{E_{ \pm}^{2} \max \left(E_{ \pm}, \delta_{S}\right)} \frac{|\vec{t}|}{T_{0}}, \\
& \left|\frac{d \overrightarrow{\boldsymbol{\epsilon}}_{\mathrm{so}}}{d\left(e V_{g}\right)} \times \frac{\overrightarrow{\boldsymbol{\epsilon}}_{\mathrm{so}}}{\left|\overrightarrow{\boldsymbol{\epsilon}}_{\mathrm{so}}\right|}\right| \approx \frac{\Gamma_{L} \Gamma_{R}}{\Delta} \frac{\Delta^{2}}{E_{ \pm}\left[\max \left(E_{ \pm}, \delta_{S}\right)\right]^{2}} \frac{|\vec{t}|}{T_{0}} .
\end{aligned}
$$

To understand why $\overrightarrow{\boldsymbol{\epsilon}}_{\mathrm{so}}$ depends on gate voltage, we study the structure of Eq. (23). We have mentioned that $\vec{\epsilon}_{\text {so }}$ is the sum of $N_{S}$ vectors characterizing spin-dependent tunneling via two different states in the dot, one being the half-filled state. The weight of each vector is determined by the energy denominators and therefore, depends on gate voltage.

If the quantum dot consists only of two levels, then change in gate voltage will modify only the modulus of $\vec{\epsilon}_{\mathrm{so}}$, i.e., the weight of the vector contribution but not the direction. It is necessary to include the contributions of at least three different levels, in order to estimate the change in the orientation of $\vec{\epsilon}_{\mathrm{so}}$. As a result, for large level spacing $\epsilon_{\perp}^{\prime}$ is reduced by a factor of $\left(\Delta / \delta_{S}\right)^{2}$. In contrast, $\epsilon_{\|}^{\prime}$ decreases slower for large level spacing, only as $\Delta / \delta_{S}$.
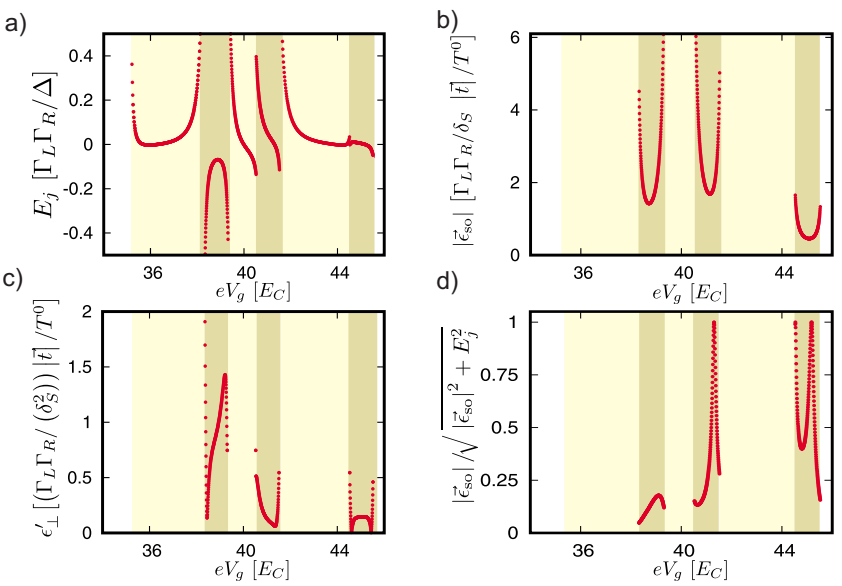

FIG. 5. (Color online) Numerical results obtained for the regime: $E_{C} / \Delta=10, \delta_{S} / E_{C}=1.5$. Six diamonds are presented: diamonds with even number of electrons are represented by lighter shading while darker shaded regions represent diamonds with odd number of electrons. For details regarding the quantities plotted, see Sec. V.

The ratio between gate-voltage sensitivity of modulus and direction of $\vec{\epsilon}_{\mathrm{so}}$ is given by $\epsilon_{\perp}^{\prime} / \epsilon_{\|}^{\prime} \approx E_{ \pm} / \max \left(E_{ \pm}, \delta_{S}\right) \leq 1$. The ratio depends on gate voltage and is expected to be largest near the middle of the Coulomb diamonds.

\section{NUMERICAL ANALYSIS}

We perform numerical calculations of the spinindependent and spin-dependent parts of the Josephson energy, based on Eqs. (22) and (23). We are particularly interested in the ratio $\left|\vec{\epsilon}_{\mathrm{so}}\right| / E_{j}$, relevant for experiments aiming to measure the separation between qubit states, and on the dependence of the direction of $\vec{\epsilon}_{\mathrm{so}}$ on gate voltage, relevant for experiments aiming to perform electrical manipulation of the qubit.

To set up the calculations, we assume that the tunneling amplitudes are independent of the lead states. We account for the fact that experimental realization of quantum dots does not permit control of the resulting localized states: we choose random energy spacing between the dot states and random absolute value of tunneling amplitudes. The parities of the localized wave functions are also chosen randomly, resulting in a random sign associated to off-diagonal spin-independent contributions $P_{m, n \neq m}^{0}\left(\epsilon_{L}, \epsilon_{R}\right)$. The parameters characterizing the dot levels are the average level spacing $\delta_{S}$ and average modulus of the spin-independent and spin-dependent tunneling amplitudes, respectively, $\left\langle\left|T^{0}\right|\right\rangle$ and $\langle|\vec{t}|\rangle$. As estimated, the ratio $\left|\vec{\epsilon}_{\text {so }}\right| / E_{j}$ is proportional to $\langle|\vec{t}|\rangle /\left\langle\left|T^{0}\right|\right\rangle$ and we include this ratio in the energy unit of the spin-dependent term.

Additional parameters in the calculation are the superconducting energy gap $2 \Delta$ and the charging energy $E_{C}$. For the numerical analysis, we need to consider a finite number of levels of the quantum dot. The results presented are obtained including a number of $N=20$ quantum-dot levels.

We vary the gate voltage over a large domain, permitting observation of multiple Coulomb diamonds (see Figs. 5-8). The size of the diamonds observed is $E_{C}$ in the case of odd 

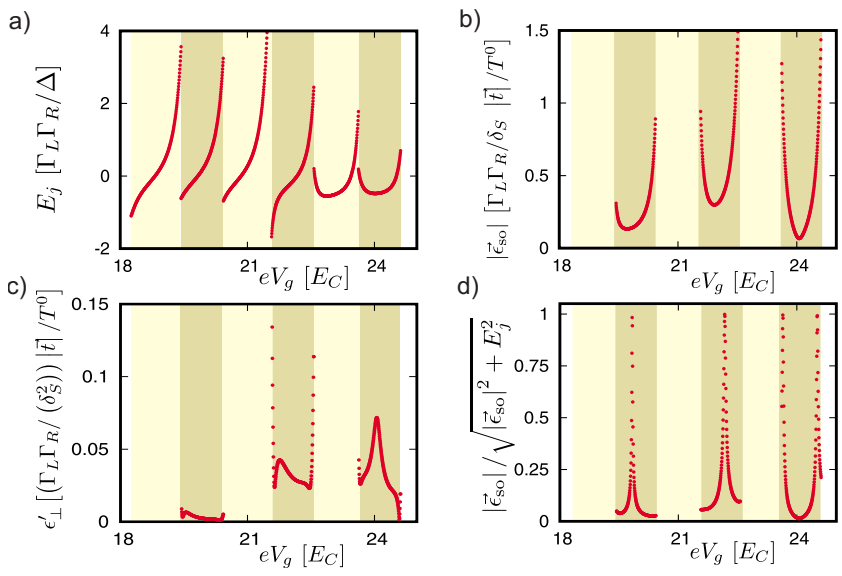

FIG. 6. (Color online) The same as in Fig. 5, for the regime: $E_{C} / \Delta=10$ and $\delta_{S} / E_{C}=0.1$.

number of electrons and increases by the level spacing in the case of diamonds with even number of electrons.

The units in Figs. 5-8 are chosen in accordance with the estimations presented in the section above, such that the value of unity corresponds to the estimated value of the quantity close to the edge, i.e., at $E_{ \pm}=\Delta$. The results confirm our estimations.

We focus on four regimes differentiated by high charging effects $E_{C} / \Delta=10$, see Figs. 5 and 6 , and relatively low charging effects $E_{C} / \Delta=1.5$, see Figs. 7 and 8. We also compare large average level spacing $\delta_{S} / E_{C}=1.5$, see Figs. 5 and 7 , to regimes where the level spacing is smaller $\delta_{S} / E_{C}=0.1$, see Figs. 6 and 8.

A general feature of the results in Figs. 5-8 is that both spin-independent and spin-dependent terms in the Josephson energy increase as we approach edges of the diamond. Furthermore, comparing Fig. 7(a), where $E_{C} \gtrsim \Delta$, with Fig. 5(a), where $E_{C} \gg \Delta$, we can conclude that for lower charging energies, the ratio between Josephson energy in the middle of diamond and energy at the edge is reduced, in agreement with our estimations. In Figs. 7 and $8(\mathrm{a}), E_{j}$ is shown to decrease in modulus toward the edge. This an unusual behavior that can be explained if there is a point in the higher-
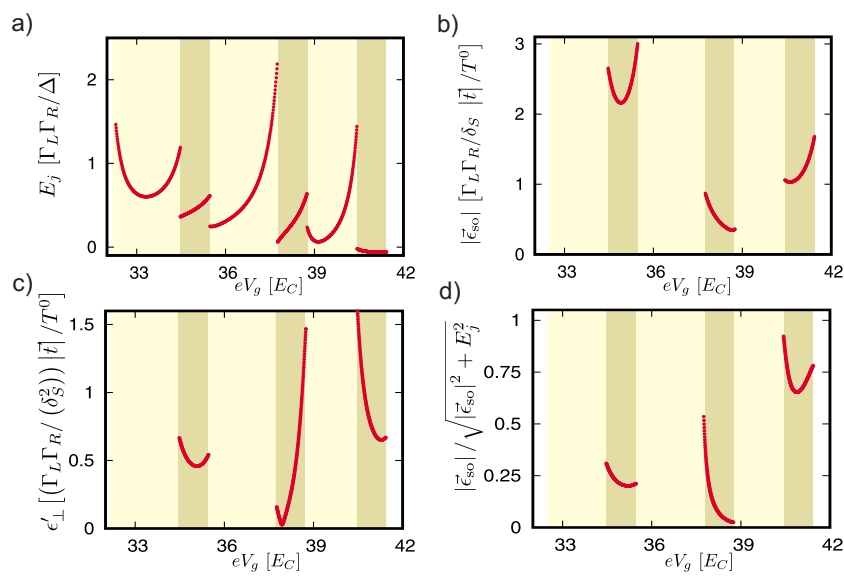

FIG. 7. (Color online) The same as in Fig. 5, for the regime: $E_{C} / \Delta=1.5$ and $\delta_{S} / E_{C}=1.5$.
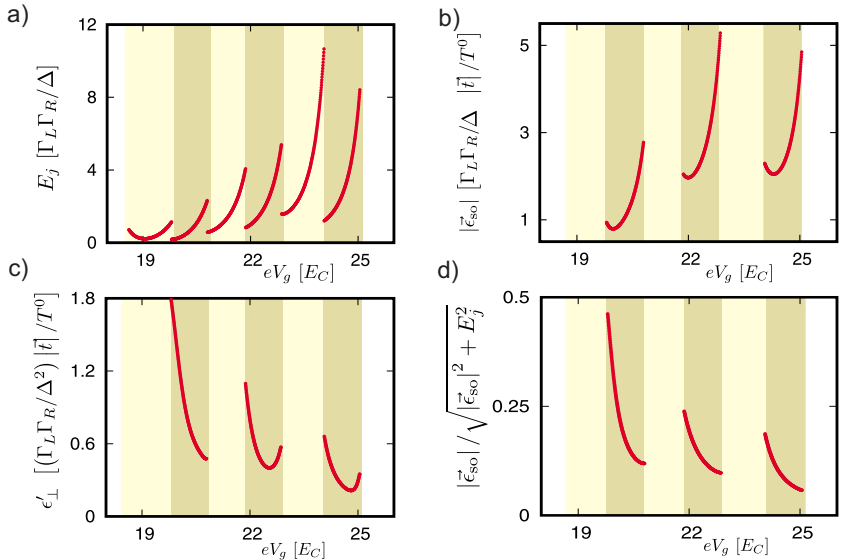

FIG. 8. (Color online) The same as in Fig. 5, for the regime: $E_{C} / \Delta=1.5$ and $\delta_{S} / E_{C}=0.1$.

energy state of the bistable region $E_{ \pm}<\Delta$, where $E_{j}$ would change sign.

Let us focus on the spin-independent contribution to the Josephson energy, presented in panel (a) of Figs. 5-8. In the single-level regime, we expect $E_{j}$ to be positive for diamonds with even number of electrons and negative for odd occupancy, as explained in Ref. 29. This behavior, observed in Fig. 5(a) in the first two diamonds, would dominate for $\delta_{S} / E_{C} \gg 1$. In the regimes presented, an interesting feature occurs: there are regions of gate voltage where $E_{j}$ changes sign within a single diamond (point of supercurrent reversal ${ }^{29}$ ). As a result, $E_{j}$ is dramatically suppressed. In these regions, $\left|\vec{\epsilon}_{\mathrm{so}}\right|$ may provide the dominating contribution to the total Josephson energy. This can be observed in panel (d) when $\left|\vec{\epsilon}_{\mathrm{so}}\right| /\left.\sqrt{E_{j}^{2}+\mid \vec{\epsilon}_{\mathrm{so}}}\right|^{2} \approx 1$, see Figs. 5 and 6. Comparing the different regimes, we observe that regions where $E_{j}$ changes sign are more likely to appear if $\delta_{S} \approx E_{C}$. In addition, the larger variations in the Josephson energy observed for $E_{C} \gg \Delta$ compared to the regime $E_{C} \gtrsim \Delta$, further increase the probability of sign reversal.

Turning to the polarization vector $\vec{\epsilon}_{\text {so }}$, panels (b) and (c) of Figs. 5-8 present its modulus and the variation in its orientation as a function of gate voltage. The modulus $\left|\vec{\epsilon}_{\mathrm{so}}\right|$ exhibits similar behavior as the absolute value of the spinindependent term $E_{j}$. In contrast to $E_{j}$, we do not observe regions of gate voltage where $\left|\vec{\epsilon}_{\mathrm{so}}\right|$ vanishes. It is not surprising: the probability that all three components of the polarization vector would vanish at the same value of gate voltage is very small.

In panel (d) of Figs. 5-8, we plot the normalized ratio between the spin-dependent and spin-independent terms $\left|\vec{\epsilon}_{\mathrm{so}}\right| / \sqrt{E_{j}^{2}+\left|\vec{\epsilon}_{\mathrm{so}}\right|^{2}}$. We note that the ratio increases in the vicinity of regions where $E_{j}$ vanishes, as is the case in Figs. 7 and $8(\mathrm{~d})$. These regions are exceptional. The common case is represented by diamonds such as diamond 1 in Fig. 5(d), where one observes a maximum of the ratio in the middle of the Coulomb diamond and decrease toward the edges. This is in agreement with our estimations, reflecting that $E_{j}$ diverges faster than $\left|\vec{\epsilon}_{\mathrm{so}}\right|$ as we approach the diamond edge.

Let us focus on the direction of $\vec{\epsilon}_{\mathrm{so}}$ and its dependence on gate voltage. We study the derivative with respect to gate 
voltage, projected in the perpendicular direction to $\vec{\epsilon}_{\mathrm{so}}$. We use the same notation as previously $\epsilon_{\perp}^{\prime}=\left|\frac{d \vec{\epsilon}_{\mathrm{so}}}{d\left(e V_{g}\right)} \times \frac{\vec{\epsilon}_{\mathrm{so}}}{\left|\vec{\epsilon}_{\mathrm{so}}\right|}\right|$.

As a general feature, the derivative is significantly reduced in the middle of the diamonds, as compared to the edge. It is interesting to note that the change in the perpendicular projection is not as smooth as the variation in $\vec{\epsilon}_{\text {so }}$ with gate voltage. In the regime $\Delta \approx \delta_{S}$, see Figs. 5,6 , and 7(c), we find regions of gate voltage where the perpendicular derivative either vanishes or abruptly changes its behavior. These features can be explained as follows. In this regime, $N_{S} \approx 1$ near the edges, meaning that the direction of $\vec{\epsilon}_{\text {so }}$ is dominated by contributions of few levels. It is possible to find regions of gate voltage where the weight of the dominating contribution vanishes, similar to the case already discussed for $E_{j}$. In the vicinity of such points, the weight of the dominating contribution changes sign. This is represented by the sharp turning points observed in Figs. 5, 6, and 7(c). In comparison, the case $\Delta \gg \delta_{S}$ presented in Fig. 8(c) shows that the behavior is smooth. In this regime, the contribution is dominated by terms of multiple levels, reducing the probability to encounter values of the gate voltage where the dominating contribution to the perpendicular derivative vanishes.

We may also conclude that $\overrightarrow{\boldsymbol{\epsilon}}_{\mathrm{so}}$ varies mainly laterally when the gate voltage is set to the middle of the diamond. Here, the variation in $\left|\vec{\epsilon}_{\mathrm{so}}\right|$ vanishes. As the gate voltage is tuned toward the diamond edges, the lateral variation is overcome by the faster divergence of $\left|\vec{\epsilon}_{\mathrm{so}}\right|$.

\section{CONCLUSION}

In conclusion, we have outlined our proposal of superconducting spin qubit. Such a unit would combine the natural representation of two-level system in terms of electron spin and advantages of superconducting qubits. Spin and superconducting qubits can be operated within the circuit, the flux and spin degrees of freedom can be easily entangled. We have demonstrated feasibility of all electric manipulation of superconducting spin qubits and more complicated quantum gates made of such qubits. Although this has been achieved previously for superconducting qubits, ${ }^{24}$ realizing the same controllability for spin qubits is remarkable.

We have discussed relaxation and decoherence of the qubit. Our analysis shows that the dominating decoherence process is typically the coupling between qubit-level spacing and magnetic-flux noise. We show that in comparison to other superconducting qubits, superconducting spin qubits have lower sensitivity to flux noise and therefore longer decoherence times. This is an important advantage in view of realizing a large number of coherent quantum operations required by quantum computation schemes.

The microscopic analysis presented shows that the spindependent part of the Josephson energy can be made sufficiently large, at least for semiconducting devices where spinorbit interaction is intrinsically strong. We predict spontaneous breaking of time-reversal symmetry in the loops containing superconducting spin qubits.

\section{ACKNOWLEDGMENTS}

We acknowledge fruitful discussions with L. P. Kouwenhoven, H. Keijzers, and S. Frolov. This work is part of the research program of the Stichting FOM.
${ }^{1}$ A. M. Nielsen and I. L. Chuang, Quantum Computation and Quantum Information (Cambridge University Press, Cambridge, 2000).

${ }^{2}$ P. W. Shor, SIAM J. Comput. 26, 1484 (1997).

${ }^{3}$ L. K. Grover, Phys. Rev. Lett. 79, 325 (1997).

${ }^{4}$ D. Loss and D. P. DiVincenzo, Phys. Rev. A 57, 120 (1998).

${ }^{5}$ R. Hanson, B. Witkamp, L. M. K. Vandersypen, L. H. Willems van Beveren, J. M. Elzerman, and L. P. Kouwenhoven, Phys. Rev. Lett. 91, 196802 (2003).

${ }^{6}$ J. R. Petta, A. C. Johnson, J. M. Taylor, E. A. Laird, A. Yacoby, M. D. Lukin, C. M. Marcus, M. P. Hanson, and A. C. Gossard, Science 309, 2180 (2005).

${ }^{7}$ F. H. L. Koppens, D. Klauser, W. A. Coish, K. C. Nowack, L. P. Kouwenhoven, D. Loss, and L. M. K. Vandersypen, Phys. Rev. Lett. 99, 106803 (2007).

${ }^{8}$ F. H. L. Koppens, K. C. Nowack, and L. M. K. Vandersypen, Phys. Rev. Lett. 100, 236802 (2008).

${ }^{9}$ S. Amasha, K. MacLean, I. P. Radu, D. M. Zumbühl, M. A. Kastner, M. P. Hanson, and A. C. Gossard, Phys. Rev. Lett. 100, 046803 (2008).

${ }^{10}$ L. Childress, M. V. G. Dutt, J. M. Taylor, A. S. Zibrov, F. Jelezko, J. Wrachtrup, P. R. Hemmer, and M. D. Lukin, Science 314, 281 (2006).

${ }^{11}$ R. Hanson, V. V. Dobrovitski, A. E. Feiguin, O. Gywat, and D.
D. Awschalom, Science 320, 352 (2008).

${ }^{12}$ P. Neumann, N. Mizuochi, F. Rempp, P. Hemmer, H. Watanabe, S. Yamasaki, V. Jacques, T. Gaebel, F. Jelezko, and J. Wrachtrup, Science 320, 1326 (2008).

${ }^{13}$ P. Cappellaro, L. Jiang, J. S. Hodges, and M. D. Lukin, Phys. Rev. Lett. 102, 210502 (2009).

${ }^{14}$ J. M. Elzerman, R. Hanson, L. H. Willems van Beveren, B. Witkamp, L. M. K. Vandersypen, and L. P. Kouwenhoven, Nature (London) 430, 431 (2004).

${ }^{15}$ F. H. L. Koppens, C. Buizert, K. J. Tielrooij, I. T. Vink, K. C. Nowack, T. Meunier, L. P. Kouwenhoven, and L. M. K. Vandersypen, Nature (London) 442, 766 (2006).

${ }^{16}$ M. Atatüre, J. Dreiser, A. Badolato, A. Högele, K. Karrai, and A. Imamŏglu, Science 312, 551 (2006).

${ }^{17}$ J. Berezovsky, M. H. Mikkelsen, N. G. Stoltz, L. A. Coldren, and D. D. Awschalom, Science 320, 349 (2008).

${ }^{18}$ R. Hanson and D. D. Awschalom, Nature (London) 453, 1043 (2008).

${ }^{19}$ T. Yamamoto, Yu. A. Pashkin, O. Astafiev, Y. Nakamura, and J. S. Tsai, Nature (London) 425, 941 (2003).

${ }^{20}$ Yu. A. Pashkin, T. Yamamoto, O. Astafiev, Y. Nakamura, D. V. Averin, and J. S. Tsai, Nature (London) 421, 823 (2003).

${ }^{21}$ R. McDermott, R. W. Simmonds, M. Steffen, K. B. Cooper, K. Cicak, K. D. Osborn, S. Oh, D. P. Pappas, and J. M. Martinis, 
Science 307, 1299 (2005)

${ }^{22}$ M. Steffen, M. Ansmann, R. C. Bialczak, N. Katz, E. Lucero, R. McDermott, M. Neeley, E. M. Weig, A. N. Cleland, and J. M. Martinis, Science 313, 1423 (2006).

${ }^{23}$ J. H. Plantenberg, P. C. de Groot, C. J. P. M. Harmans, and J. E. Mooij, Nature (London) 447, 836 (2007).

${ }^{24}$ I. Chiorescu, Y. Nakamura, C. J. P. M. Harmans, and J. E. Mooij, Science 299, 1869 (2003).

${ }^{25}$ J. M. Martinis, K. B. Cooper, R. McDermott, M. Steffen, M. Ansmann, K. D. Osborn, K. Cicak, S. Oh, and D. P. Pappas, R. W. Simmonds, and C. C. Yu, Phys. Rev. Lett. 95, 210503 (2005).

${ }^{26}$ L. I. Glazman and K. A. Matveev, JETP Lett. 49, 659 (1989).

${ }^{27}$ V. A. Khlus, A. V. Dyomin, and A. L. Zazunov, Physica C 214, 413 (1993).

${ }^{28}$ Y. J. Doh, J. A. van Dam, A. L. Roest, E. P. A. M. Bakkers, L. P. Kouwenhoven, and S. De Franceschi, Science 309, 272 (2005).

${ }^{29}$ J. A. van Dam, Yu. V. Nazarov, E. P. A. M. Bakkers, S. De Franceschi, and L. P. Kouwenhoven, Nature (London) 442, 667 (2006).
${ }^{30}$ N. M. Chtchelkatchev and Yu. V. Nazarov, Phys. Rev. Lett. 90, 226806 (2003).

${ }^{31}$ A. Zazunov, R. Egger, T. Jonckheere, and T. Martin, Phys. Rev. Lett. 103, 147004 (2009).

${ }^{32}$ B. Béri, J. H. Bardarson, and C. W. J. Beenakker, Phys. Rev. B 77, 045311 (2008).

${ }^{33}$ J. Danon and Yu. V. Nazarov, Phys. Rev. B 80, 041301(R) (2009).

${ }^{34}$ A. Ekert and R. Josza, Rev. Mod. Phys. 68, 733 (1996).

${ }^{35}$ G. Ithier, E. Collin, P. Joyez, P. J. Meeson, D. Vion, D. Esteve, F. Chiarello, A. Shnirman, Y. Makhlin, J. Schriefl, and G. Schön, Phys. Rev. B 72, 134519 (2005).

${ }^{36}$ S. Nadj-Perge, S. Frolov, J. van Tilburg, J. Danon, Yu. V. Nazarov, R. Algra, E. Bakkers, and L. Kouwenhoven, arXiv:1002.2120 (unpublished).

${ }^{37}$ R. Bauernschmitt, J. Siewert, Yu. V. Nazarov, and A. A. Odintsov, Phys. Rev. B 49, 4076 (1994).

${ }^{38}$ A. V. Rozhkov, D. P. Arovas, and F. Guinea, Phys. Rev. B 64, 233301 (2001). 